\title{
Generate a 3D CAD models for Pose Estimation
}

\author{
Elmi Abu Bakar, Tetsuo Miyake, Takashi Imamura and Zhang Zhong, Member, IEEE
}

\begin{abstract}
The pose estimation of 3D object of products in production line is needed beforehand. In order to perform shape measurement of objects corresponding to speed of the mass production lines before the contact measurement is done, the information of shape representation and matching of object is required. Objects are compared with its descriptor. The descriptor conceptually subtracted from each other to form a scalar metric. If the value difference is smaller, the object shape is considered closer to each other. The rotation object from its original pose can produce the information to estimate its rotation pose by using the boundary. In this paper, we proposed the method to measure 3D object pose from the designing stage of 3D CAD data.
\end{abstract}

Index Terms-CAD, Boundary Descriptor, Shape Classification, Euclidean distance, Correlation.

\section{INTRODUCTION}

In manufacturing industry, Computer Aided Testing (CAT) is the latest important technology. It's because CAT exists in a final stage in following flow of manufacturing: 1.Design 2.Production 3.Quality Control by Testing. This technology had been developed in order to overcome the former method by 3D Measuring Instrument that consume a lot of time for examine one object. If the object position/pose is known in advance before measurement, much time can be reduced. Moreover if the system is constructed by using comparatively inexpensive methods, the complete testing can be conducted in many production lines such as casting, molding and press. Moreover, the image based measurement method promise high accuracy .The method is using the shape description since it's known as robustness, compactness, low computation complexity and perceptual similarity measurement. Using image based retrieval method we can estimate the pose by optimizing the error between visual input and the projection of the pose to image space. Usually, image features are extracted from visual input to allow more efficient matching. In many literatures, features are often used as edges, silhouettes, color and motion. We focus on edges because they can be easily extracted from image. From this edge, we investigate in details about boundary of object in that image.

In order to that, we need to look on detection of that boundary. In image processing topics, boundary detection is most fundamental problems. Until now many methods have

Manuscript received Sept 23, 2009. This work was supported in part by Global COE Program "Frontiers of Intelligent Sensing ”, MEXT, Japan.

Elmi Abu Bakar, Tetsuo Miyake, Takashi Imamura and Zhang Zhong is with the Toyohashi University of Technology, Tenpaku Hibarigaoka 1-1, Toyohashi Aichi, Japan, email:elmi@ieee.org, (miyake,ima,zhang) @is.pse.tut.ac.jp been introduced in the engineering literature. The object image can be understood from boundary information or region information in practical image. In $3 \mathrm{D}$ view of object image we can understand this information through projection system or designing a 3D object through AutoCAD platform. If we can understand the image information through boundary by calculating according some descriptor, surely we can compute the shape of any object easily without pre information beforehand. In previous studies, image feature such as intensity, texture, color, are viewed as boundary [1]. Thus, a variety of operators using the first derivative of the gray scale or texture can be used to detect this discontinuity. To obtain the accuracy of boundary, a refinement of boundary on basis of initial plan is necessary. Statistical methods can also be introduced in boundary detection [2]. This method has modeled the boundary as a deterministic signal and the noise as random signal with distribution function. Other approaches to boundary detection involve the use of transformation. Transformation is including scale change, translation and rotation. Boundary of object can be understood detail after some process of description. The most well known descriptor works on outline or boundary of object such as Fourier descriptor. Fourier descriptor itself fulfilled some criteria of shape representation. Basically, common criteria such invariance to translation, scale, rotation and symmetric transformations, scalability and efficiency, uniqueness and the most is robustness to distortion and noise $[1,4]$. Above criteria should be fulfilled for shape representation for reliable shape retrieval and matching [3].

In this paper, we proposed the method to measure $3 \mathrm{D}$ object pose from the designing stage of $3 \mathrm{D}$ CAD data into calculation of 3D object feature matching using MATLAB platform for friendly user into production inspection system. In experimental results, we show the effectiveness of this method to provide the information to non-contact measurement based on CAD models data. Our proposed method uses the boundary estimation of CAD data by Fourier Descriptor in order to create image information of shape before it can be using for pose estimation in Computer Aided Testing System.

This paper constituted as follows. Section II, we explain our model design of object using 3D design modeler. Our feature extraction of object is in section III. Section IV, feature classification by boundary based using Fourier descriptor and correlation. The experimental results are shown in section V and we discuss in section VI. Finally the conclusion of the paper is shown in section VII.

\section{Designing Stage}

This section describes the model design of CAD data, section A explains the modeler for 3D model built 
environment and in section B the interface platform to read from 3D model data into MATLAB environment and rendering projection for model database.

\section{A. Model design}

In this work we use Solid Works [7], a well-known solid modeling CAD system to perform CAD related operation Solid Works uses a feature-based "parametric" approach to modeling and assembling. In the Solid Works 3D modeling environment the creation of a solid or surface typically begins with the definition of topology in either a $2 \mathrm{D}$ or $3 \mathrm{D}$ sketch. The topology defines the connectivity and certain geometric relationships between vertices and curves both in the sketch and external to the sketch. To this topology are added dimensions, which determine the lengths and sizes for the curves and locations for the vertices in conjunction with topological constraints.

The dimensions, which are added, are termed "parameters" because they can be changed either independently or by "parameters" created prior to their creation. Though, Solid Works as our tool to design the model, other tools such Pro-E, Catia or others also can be used for design according the familiarity of tool application.

In our research we first design our CAD object model by using this $3 \mathrm{D}$ modeler. We design from $2 \mathrm{D}$ view into $3 \mathrm{D}$ view. Since the Solid Works provide an interfacing to the system, so that we are relieved from tedious programming work, such as reading CAD files and identifying individual surfaces. A model typically consists of several individual surfaces, which were generated during the design process. In our research we focused only to generate the $3 \mathrm{D}$ model and transform into image range. Our designing stage of research model is considered in a few aspects. First, the aims of model should fulfill the criteria of casting goods that obey the accuracy in shape dimension, corner curve and internal holes existence.


Fig. 1. 3D design models

\section{B. Rendering the models}

Data that we created with 3D modeler is saved in stereo lithography (STL) format. STL file describes a raw unstructured triangulated surface by the unit normal and vertices of triangles using a three dimensional Cartesian coordinate system. This format preserved the important information of model that can easily generate model back when it considered necessary. We built the environment using MATLAB program. The model transformation and rendering is applicable by using this algorithm. The projection parameter to view the image is using default parameter of rendering in graphic tools. In Fig. 1 we describe the CAD Models in our database for rendering purpose.

The result of rendering of CAD data into MATLAB platform image has been showed in Fig.2. In order to plot the model as Fig.2 shown, first we defined the related transformation matrix in our homogenous coordinate system.
With this derivation of transformation we easily parameterize the camera position, orientation, focal length and scaling and translation parameters in the image plane.

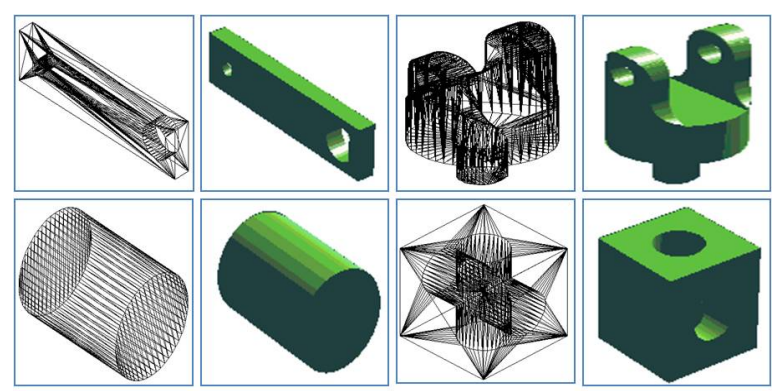

From STL format into Rendering in MATLAB

Fig. 2. Matlab Rendering

The illumination using camera light with specific parameter also being applied in the rendering system with consideration of azimuthally and orientation angle of the model view. The actual camera rendering of the CAD model is according to camera parameter from the projection system. In addition, the object is plotted in perspective projection. Fig.3, shows the rotation angle for rendering the model.

\section{FEATURE EXTRACTION}

As for the CAD model, the feature of edge also has been processed for image processing to obtain edge image. The input image is model rendering of CAD model. Before we can achieve correct edge detection it is necessary to consider a noise problem to our system. In actual practice of projection often image is projected containing noise. Below section we explain in the details.
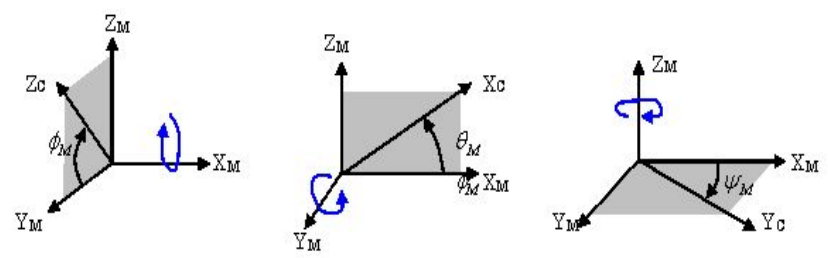

Fig. 3. Rotation Angle

\section{A. Noise sensitivity}

Digital images are sensitive to noise. Noise is the result of errors in the image acquisition process that result in pixel values that do not reflect true intensities of real scene. For example, if the image is acquired directly in a digital format, the mechanism for gathering the data (such as a CCD camera) can introduce noise [5]. For this reason, in order to ensure our algorithm is noise sensitive or not we do apply some noise into rendering image that we produce in chapter III in our preprocessing calculation. In our simulation of object in rotation of 3 axial, we found some rotation pose happen some noise that influence by illumination in rendering system. Also, we added salted noise together with that illumination reflection at object corner to qualify our preprocessing algorithm is suitable in actual condition of projection image.

Here, we made some combination of noise which consist of illumination noise and salted noise in condition which salted noise value at 0.05 and high brightness illumination. In order to remove the noise and to get the smooth outline boundary, here we apply the Histogram Equalization threshold selection on image and to contour the boundary 
image using Adaptive threshold detector [4]. This noise generation is done in order to understand a real scene of projection in real world.

\section{B. Image processing}

The preprocessing of image is done to extract the input image into boundary image or contour image before we calculate the shape description of object. The outline silhouette we choose in our research can be described as single closed curve. The result of the preprocessing is shown in Fig. 4.

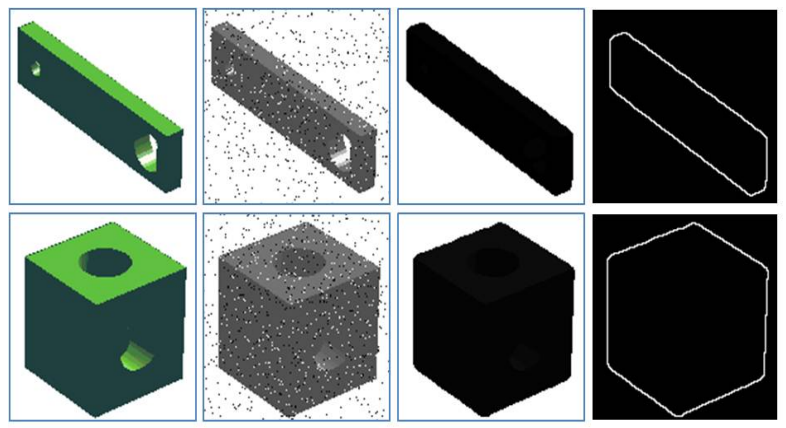

Rendering Image into Boundary Image

Fig. 4. Preprocessing

The binarization using Histogram Equalization method and edge extraction are performed in the input image using Adaptive threshold. The preprocessing is calculated by using MATLAB environment.

\section{Applying Fourier Transform}

The discrete Fourier Transform of $\mathrm{s}(\mathrm{k})$ gives

$$
a(u)=\frac{1}{N} \sum_{k=0}^{N-1} s(k) e^{\frac{-2 u x \pi}{N}}
$$

For $\mathrm{u}=0,1,2, \ldots, \mathrm{N}-1$. The complex coefficients $\mathrm{a}(\mathrm{u})$ are called Fourier descriptors of the boundary. Applying inverse Fourier Transform to a $(\mathrm{u})$ restores $\mathrm{s}(\mathrm{k})$

$$
s(k)=\sum_{u=0}^{N-1} a(u) e^{\frac{2 u x \pi}{N}}
$$

For $\mathrm{k}=0,1,2, \ldots, \mathrm{N}-1$. The restored pixel values are exactly the same as the ones that we started with. This coefficients also called Fourier descriptors, describe the shape of the object in frequency domain. The transformation is loss-less since the energy in the frequency domain is the same as the energy in the spatial domain (due to Parseval's thereom) and also the inverse Fourier Transform gives the original boundary function.

\section{Boundary Descriptor}

Let us consider $\mathrm{N}$ points in digital boundary of the shape on $\mathrm{x}$ y-plane. Traveling the boundary clockwise keeping constant $\mathrm{N}$ seconds and Keep the coordinate every second. So, we have complete set of coordinates describing the boundary. The complex coordinates of the complete boundary can be written as;

$$
s(k)=x_{k}+i y_{k}
$$

For $\mathrm{k}=0,1,2, \ldots, \mathrm{N}-1$. which is periodic with $\mathrm{N}$. As stated in above equation, the coordinate system where $\mathrm{x}$-axis treated as real axis and the $y$-axis as imaginary axis of a sequence of complex numbers.

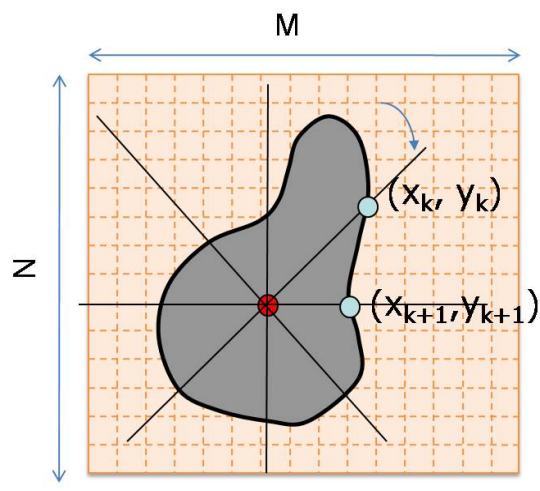

Fig. 5. Boundary Image

\section{FeAture ClassificAtion}

1) Shape matching using Euclidean distance:

To match the boundary image from database, the representation of edge using above equation can be considered as the boundary function.

$$
\begin{array}{r}
s(k)=x_{k}+i y_{k} \\
s^{\prime}(k)=x_{k}{ }^{\prime}+i y_{k}{ }^{\prime}
\end{array}
$$

(For $\mathrm{k}=0, \ldots, \mathrm{N}-1$ ). A typical measure of similarity between boundaries in Euclidean distance, which corresponds to mean error and which is also directly related to the cross correlation.

$$
\operatorname{Dist}\left(s, s^{\prime}\right)=\sqrt{\sum_{f=0}^{N-1}\left|S_{F}-S^{\prime}{ }_{F}\right|^{2}}
$$

2) Shape matching using Image Correlation:

Typically shape matching measures include cross correlation. Sum of

For $\mathrm{k}=0,1,2, \ldots, \mathrm{N}-1$. The restored pixel values are exactly the same as the ones that we started with. This coefficients also called Fourier descriptors, describe the shape of the object in frequency domain. The transformation is loss-less since the energy in the frequency domain is the same as the energy in the spatial domain (due to Parseval's theorem) and also the inverse Fourier Transform gives the original boundary function. Sum of absolute differences and the correlation coefficient is usually evaluated mathematically the below equation (5). Consider a digital image m' ( $i, j$ ) of size M X N, we determine a region similar to some region $\mathrm{m}(\mathrm{i}, \mathrm{j})$ of size $\mathrm{M} X \mathrm{~N}$. In Fig. 5 shows the boundary points for indexing the edge image. Here, we perform the correlation between $m^{\prime}(i, j)$ and $m(i, j)$ by this where $\bar{m}^{\prime}$ and $\bar{m}$ is the mean intensity of image $\bar{s}^{\prime}$ and $\bar{s}$. The above equation, in facts, represents the ratio between covariance of the two images and the product of their respective standard deviation. Under certain statistical assumptions, the value measured by the correlation 
coefficient gives linear indication of similarity between images. This is useful in order to quantitatively measure confidence or reliability in a match.

$$
\operatorname{Corr}=\frac{\sum_{j=0}^{N-1} \sum_{i=0}^{M-1}\left[m^{\prime}[i, j]-\bar{m}^{\prime} \llbracket[m[i, j]-m]\right.}{\sqrt{\sum_{j=0}^{N-1} \sum_{i=0}^{M-1}\left(m^{\prime}[i, j]-\bar{m}^{\prime}\right)^{2}} \sqrt{\sum_{j=0}^{N-1} \sum_{i=0}^{M-1}(m[i, j]-m)^{2}}}
$$

\section{EXPERIMENTAL RESULTS}

In this section, we describe a set of experiments data of CAD model image in our database for evaluating feature classification performance. Our candidate's image is in gray scale images ( $560 \times 420$ pixels). The image taken in range of $[0,360]$ for each 1 degree one pose in rotation of $\mathrm{Z}$ axis of geometrical system coordinate. Preprocessing the image as mentioned in previous section and calculating the image boundary feature vector in feature classification and feature matching. In order to detail understanding of the characteristic each model of object while rotation is performed the feature matching toward clock wise is considered in this calculation.

Table I

CANDIDATES IMAGE

\begin{tabular}{cl}
\hline \hline Rotation & Angle Value \\
\hline Z Axis & {$[0,1,2,3, \ldots, 359,360]$} \\
\hline \hline
\end{tabular}

Table II

MATCHING SETS

\begin{tabular}{cl}
\hline \hline Direction & Matching sets \\
\hline $\mathrm{CW}$ & {$[0-(0), 0-(1) \ldots, 0-(360)]$} \\
\hline \hline
\end{tabular}

In this section we present our results for model in database. The Spider object results are shown in Fig. 6 and Fig. 8 and the Crank Arm object results are shown in Fig.7 and Fig.9. In each figure, describe in two result which represent the calculation of distance using Euclidean distance and the Correlation shape matching data. The result of rotation of CW direction of each degree is in Fig. 6 and Fig.7 we plotted the noise performance for Edge Image with noise and without noise. Our processing algorithm performed well and the graph tendency show almost same. In Fig. 8 and Fig.9 we plotted two classification of results of two models. Here, the method using Euclidean distance matching shows the matching against initial pose 0 in four region for Crank Arm object and more than 4 region for Spider Object. A Reason why spider object plotted curve changing in detail because the symmetrical object characteristic. With the consideration of correlation result in same graph plotted, we are able to determine interpolation point each matching set.

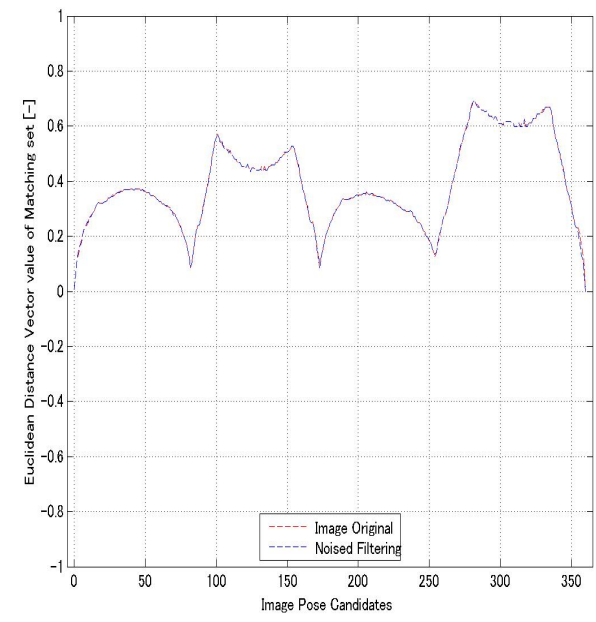

Fig. 6. Crank Arm Object Noise performance

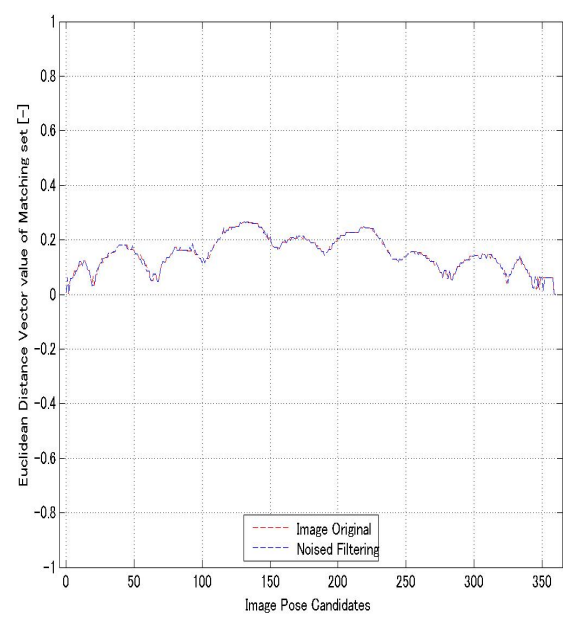

Fig. 7. Spider Object Noise performance

\section{DISCUSSION}

In our calculation of boundary edge using Fourier descriptors we found that for rotation estimation of pose against original pose show some change characteristic of every individual pose. In order to measure the correct pose of object rotation we combined two classification methods into consideration. The reason behind this, because each pose of object should bring a numerical value describing its pose.

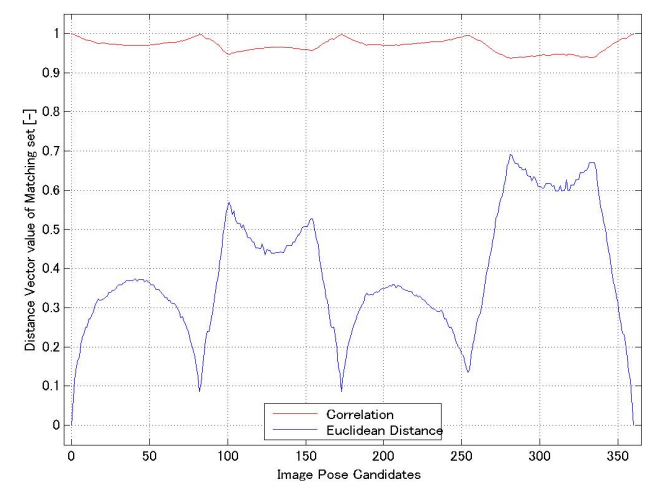

Fig. 8. Crank Arm Object Feature Classification 


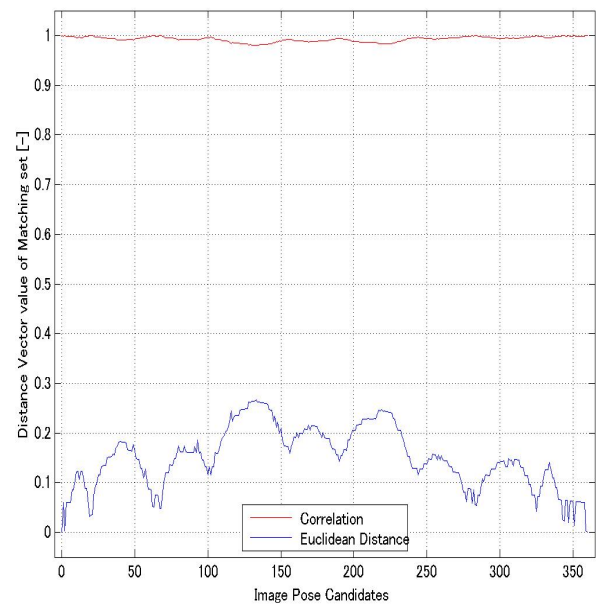

Fig. 9. Spider Object Feature Classification

\section{CONCLUSION}

In this paper, we proposed the novel method for object pose estimation using 3D CAD model information into silhouette boundary. Descriptors with number low number of coefficients we calculated using equidistant sampling. Two tests set with rotation every 1 degree for 360 poses were used. Adding noise to the silhouette boundary did not affect the recovery performance. Therefore, the proposed method is very effective in practical use. As a future subject, we will consider the actual projection image in 1-degree subsequence with complicated model shape feature classification into calculation.

\section{ACKNOWLEDGMENT}

This research was supported in part by Global COE Program "Frontiers of Intelligent Sensing ”, MEXT, Japan .

\section{REFERENCES}

[1] D.S.Zhang and G.J.Lu, "Shape Retrieval Using Fourier Descriptors." Int. Conference on Multimedia and Distance Education. Fargo, ND, USA, June 2001 .

[2] M.Sonka,V.Hlavac, R.Boyle, Image Processing, Analysis, and Machine Vision, Second Edition, Brooks /Cole Publishing Company, 1999 , pp. 240-248.

[3] J.BOHM,C.BRENNER,Others, "Automated Extraction of Features from CAD Models for 3D object recognition.” ISPRS, Vol.XXXIII, Asmeterdam, 2000.

[4] The Mathworks, Image Processing toolbox User's Guide, Version 5 , The Mathwork, Inc. Natick, MA., 1993-2005.

[5] Zahn C.T.and Roskies R.Z. "Fourier Descriptors for Plane Closed Curves". IEEE Transactions on Computers, March 1972, Vol.C21, No,pp.269-281.

[6] Hui .C, Ohnishi.N, and Others. "Retrieval-Combination Approach to estimate 3D Human Pose from Monocular Image.” ITE, 2007, Vol.61, No.2, pp223-233.

[7] Solid Works(1995) Solid works 2001 plus. USA, Massachusetts: Solid Works corporation.

[8] S.Ekvall,D.Kragic,F.Hoffman, “ Object recognition and pose estimation using color and geometric modeling." Journal of Image and Vision Computing 23, 2005, pg 943-945.

[9] A.B.Elmi, T.Miyake, Z.Zhang and T.Imamura, "Shape representation and matching for Computer Aided Testing System,Precision Engineering Japan Fall Conference", 2006, pg. 717-718.

[10] Jagadish HV. " A Retrieval technique for similar shapes". In. Proc ACM SIGMOD International Conference on Management of DATA, Denver, Colombia, USA, 1991, pp.208-217.
ELMI Abu Bakar graduated from Department of Mechanical Engineering at Kisarazu National College of Technology in 1997 and received his B.Eng from Department of Mechanical Engineering at Iwate University In 1999. He worked as Tooling Engineer in Parts Production Department of Alps (M).Co from 1999 to 2002. Joined University Science Malaysia as teaching staff for School Of Mechanical Engineering and on leave for continuing study for M.Eng and currently Phd candidate at Department of Production Systems Engineering, Toyohashi University Of Technology. His research interest includes CAD, Machine Vision and Quality Control Measurement. He is a member of Japan Society of Precision Engineering and the IEEE Instrumentation and Measurement Society.

MIYAKE Tetsuo received his B.S and M.S degrees in Agricultural Mechanical Engineering and Doctoral degree in Electrical and Electronic Engineering from Tokyo University in 1975, 1978, and 1987. He worked in the Central Research Laboratory of Asahi Glass.Co. from 1984 to 1990 . He is currently an associate professor at Toyohashi University of Technology. His research interests include non contact measurement, motion tracking, and human interface. He is member of the Information Processing Society of Japan, the Institute of Image Information and Television, the Society of Instrument and Control Engineers, and the IEEE Computer Society.

IMAMURA Takashi received his B.E. degree from National Institution for Academic Degrees in 1997. And he received the M.E. and Dr.Eng. degree from Toyohashi University of Technology, Japan in 1999 and 2003, respectively. From 2003 to 2005, he was a post-doctoral researcher in University of Paris 13th, France, and Toyohashi University of Technology, Japan, respectively. He is currently an assistant professor at Toyohashi University of Technology. His research interests include Mechanism design, Industrial Application of control engineering, Educational robotics and Measurement for Car Driver's behavior. He is member of the Japan Society of Mechanical Engineers, the Society of Instrument and Control Engineers, The Robotics Society of Japan, The Institute of Electronics, Information and Communication Engineers, Society of Automotive Engineers of Japan, and the IEEE System, Man and Cybernetics Society.

ZHANG Zhong received his Bachelor Engineering, Master Engineering degrees in 1982 and 1984, respectively, from Xi'an Highway University, China and his Doctor engineering degree in 1993 from Okayama University, Japan. He was a visiting scholar at the University of Melbourne, Australia in 1998. He engaged in research regarding intelligent system and signal, Image processing as a senior researcher at the Industrial Technology Center of Okayama Prefecture, an associate professor at Okayama Prefecture University, and now a professor at Instrumentation Systems Laboratory of Toyohashi University of Technology. He is member of the Japan Society of Mechanical Engineers, The Society of Instrument and Control Engineers, member of Research Institute of Signal Processing Japan, The Institute of Electrical Engineers of Japan, Society of Automotive Engineers of Japan, and the IEEE member. 